\title{
ОЦЕНКА ПОКАЗАТЕЛЕЙ «ОТКАЗ В ОБСЛУЖИВАНИИ» ПРИ ОРГАНИЗАЦИИ РЕМОНТНЫХ РАБОТ МНОГОКОМПОНЕНТНОГО ОБОРУДОВАНИЯ
}

\author{
(c) 2020 Ю. М. Краковский ${ }^{\bowtie 1}$, Н. А. Хоанг ${ }^{2}$ \\ ${ }^{1}$ Иркутский государственный университет путей сообщения \\ ул. Чернышевского, 15, 664074 Иркутск, Российская Федерация \\ ${ }^{2}$ Иркутский государственный аграрный университет им. А. А. Ежевского \\ n. Молодежный, 1/1, 664038 Иркутский р-он, Иркутская обл., Российская Федерация
}

\begin{abstract}
Аннотация. В данном исследовании обсуждается организация ремонтных работ сложного оборудования, предполагающая наличие страхового фонда, который выполняет две функции: накапливает платежи с различной периодичностью для выполнения различного вида ремонтных работ, а затем по мере необходимости оплачивает эти работы. Математическое описание состояния страхового фонда основано на случайном процесса риска, который используется в теории рисков. Этот подход позволил ввести численные модели показателей надежности, характеризующих отказ в обслуживании оборудования. Обслуживание связано с ремонтными работами, а отказ происходит по причине отсутствия их финансового обеспечения. Численными моделями показателей надежности являются: вероятность безотказной работы, средняя наработка, гамма-процентный ресурс, вероятность безотказной работы остаточного ресурса, средний остаточный ресурс; гамма-процентный остаточный ресурс. Для исследования этих показателей создана программа, которая, используя событийный подход, создает необходимые выборочные значения, а затем обрабатывает их численными методами. Моделирующая программа имеет возможность «настраиваться» на различные исходные данные, что позволяет проводить исследования в их широком диапазоне.

Ключевые слова: многокомпонентное оборудование, случайный процесс риска, показатели надежности, имитационное моделирование.
\end{abstract}

\section{ВВЕДЕНИЕ}

Современное автоматизированное оборудование является многокомпонентным, содержащим различные подсистемы: механическую, электрическую, электронную, информационно-управляющую и др. Это предъявляет дополнительные требования к его мониторингу и диагностике, а в дальнейшем к технологии и организации ремонтных работ.

Мониторинг состояния - это диагностика состояния оборудования во время его работы [1]. Мониторинг состояния позволяет прогнозировать, какие компоненты могут

Краковский Юрий Мечеславович e-mail: 79149267772@yandex.ru выйти из строя в ближайшее время, что, в свою очередь, позволяет предотвратить внезапный их отказ. Это способствует проводить его обслуживание и ремонт по техническому состоянию $[2,3]$.

В настоящий момент при подготовке специалистов по направлениям, связанных с автоматизацией технологических процессов и производств, средств транспорта, как правило, уделяют внимание изучению вопросов, направленных на вычисление показателей надежности ремонтируемых и неремонтируемых объектов, методам оценки надежности нерезервированных и резервированных систем, принципам конструирования оборудования, обеспечивающих получение надежных систем $[4,5]$. Меньшее внимание уделяется Контент доступен под лицензией Creative Commons Attribution 4.0 License.
The content is available under Creative Commons Attribution 4.0 License. 
Оиенка показателей «Отказ в обслуживании» при организации ремонтных работ ...

вопросам обслуживания сложного роботизированного оборудования в условиях неопределенности и ограниченных финансовых ресурсов, хотя это важное направление, связанное с мониторингом его состояния.

Здесь можно отметить работы, в которых предполагается наличие страхового фонда, который выполняет две функции $[6,7]$ :

1) накапливает платежи с различной периодичностью для выполнения различного вида ремонтных работ оборудования (пополнение страхового фонда). Такими работами являются: а) текущие; б) аварийные; в) капитальные. Для каждого вида работ устанавливается периодичность пополнения страхового фонда (сутки) и их стоимость (млн. руб.). В исследовании периодичность платежей для выполнения текущих ремонтных работ равна 10 сут., для выполнения аварийных ремонтных работ - 30 сут., для выполнения капитальных ремонтных работ - 90 сут.;

2) по мере необходимости оплачивает эти ремонтные работы (уменьшение страхового фонда). Для каждого вида работ устанавливается периодичность использования страхового фонда (сутки) и их стоимость (млн. руб.). Периодичности использования страхового фонда являются случайными величинами с известными функциями распределения и параметрами.

В работах $[6,7]$ для математического описания состояния страхового фонда в момент времени $t$ предлагается использовать случайный процесс риска - $R(t)$. Возможность применения случайного процесса риска в качестве математической модели описания страхового фонда основана на применимости этого процесса в теории рисков [8]).

\section{МАТЕМАТИЧЕСКОЕ ОПИСАНИЕ СТРАХОВОГО ФОНДА}

Следуя работе [6] и учитывая три вида ремонтных работ, определим случайный процесс риска для описания состояния страхового фонда следующим образом

$$
\begin{aligned}
R(t) & =X_{0}+Y 1(t)+Y 2(t)+Y 3(t)- \\
& -Y A(t)-Y T(t)-Y K(t),
\end{aligned}
$$

где $X_{0}$ - начальные средства страхового фонда; $Y j(t)$ - суммарные накопления платежей по видам работ к моменту времени $t$, $(j=1,2,3) ; \quad Y A(t)$ - суммарные затраты по аварийным ситуациям; $Y T(t)$ - суммарные затраты для выполнения текущих работ; $Y K(t)$ - суммарные затраты для выполнения капитальных работ. Описание компонент процесса (1) приведено в работе [6].

Учитывая, что процесс (1) предлагается исследовать с применением имитационного моделирования, в табл. 1 приведены законы распределения и их числовые характеристики для интервалов времени между видами работ и затратам на эти работы: $m$ - математическое ожидание, $k_{v}$ - коэффициент вариации. Выбор вероятностных моделей осуществляется на основе литературных рекомендаций из теории надежности и рискового страхования [5, 9-11].

Для случайного процесса риска (1) определяется момент времени $\tau$, когда первый раз выполняется условие $R(t)<0$,

$$
\tau=\min _{t}\{t: R(t)<0\} .
$$

Величина (2) в работе [6] рассматривается как ресурсо-затратный риск, так как он оценивает модель «Ресурсы-затраты» для ремонтных работ, связанных с процессом экс-

\begin{tabular}{|c|c|c|c|c|}
\hline Работы & \multicolumn{2}{|c|}{ Интервалы времени } & \multicolumn{2}{|c|}{ Затраты } \\
\hline \multirow[t]{2}{*}{ Текущие } & \multicolumn{2}{|c|}{ Бирнбаума - Саундерса } & \multicolumn{2}{|c|}{ Логнормальное } \\
\hline & $m=15,0$ & $k_{v}=0,20$ & $m=3,5$ & $k_{v}=0,20$ \\
\hline \multirow[t]{2}{*}{ Аварийные } & \multicolumn{2}{|c|}{ Вейбулла } & \multicolumn{2}{|c|}{ Парето } \\
\hline & $m=45,0$ & $k_{v}=0,30$ & $m=10,0$ & $k_{v}=1,50$ \\
\hline \multirow[t]{2}{*}{ Капитальные } & \multicolumn{2}{|c|}{ Гамма } & \multicolumn{2}{|c|}{ Нормальное } \\
\hline & $m=60,0$ & $k_{v}=0,25$ & $m=20,0$ & $k_{v}=0,15$ \\
\hline
\end{tabular}

Таблииа 1. Вероятностные модели и их числовые характеристики

[Table 1. Probabilistic models and their numerical characteristics] 


\section{Ю. М. Краковский, Н. А. Хоанг}

плуатации сложного оборудования. Разность между ресурсами и затратами должна быть положительной, иначе возникает риск невыполнения этих работ.

В результате многократного имитационного моделирования процесса (1), для величин (2) создается выборка объема $n$, где

$$
0<\tau \leq T_{M} .
$$

Здесь $T_{м}-$ максимальное время создания реализации процесса (1) при имитационном моделировании. Число промоделированных реализаций равно $n_{0}$, оно больше объема выборки. В проводимом исследовании $n_{0}=20000$, а объем выборки $n$ определяется величиной $T_{м}$.

В результате имитационного моделирования получается выборка

$$
T=\left(\tau_{1}, \ldots, \tau_{i}, \ldots, \tau_{n}\right) .
$$

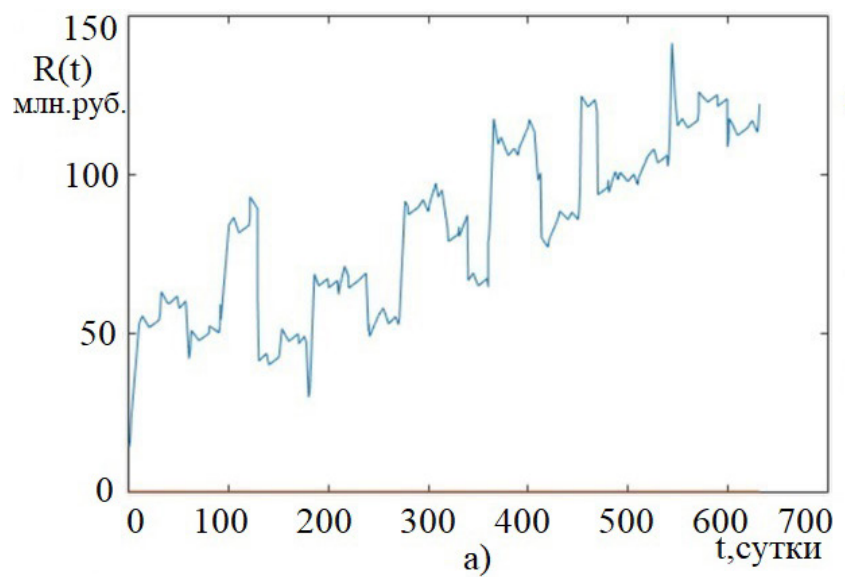

Моделирующая программа, реализующая процесс (1), использует событийный подход, ее описание можно посмотреть в работе [12]. Моделирующая программа имеет возможность «настраиваться» на различные исходные данные, что позволяет проводить исследования в их широком диапазоне.

На рис. 1 показаны возможные реализации процесса (1), полученные при имитационном моделировании. На рис. 1-а приведен вариант, когда за время $T_{\text {м }}$ процесс (1) не пересекает ось времени (выборочное значение (3) не создается); на рис. 1-б приведен вариант, когда выборочное значение создается. Соотношение между $n_{0}$ и $n$ зависит от исходных данных. На рис. 2 приведены гистограммы частот для выборки (4). На рис. 2-а она приведена для 180 суток, а на рис. 2-б для

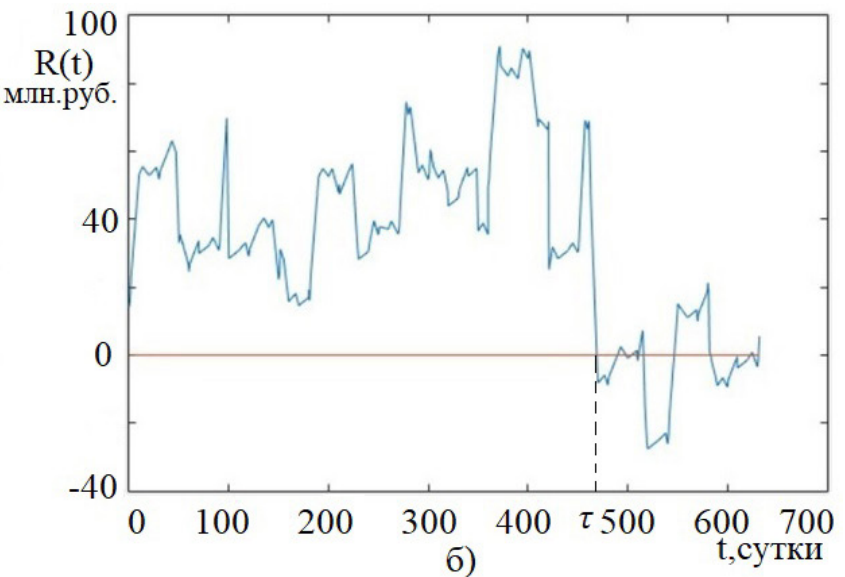

Рис. 1. Графическое представление случайного процесса риска (1):

а - когда выборочное значение отсутствует, б-когда выборочное значение создается [Fig. 1. A graphical representation of a random risk process (1):

$a$ - when there is no sample value, $b$ - when the sample value is created]
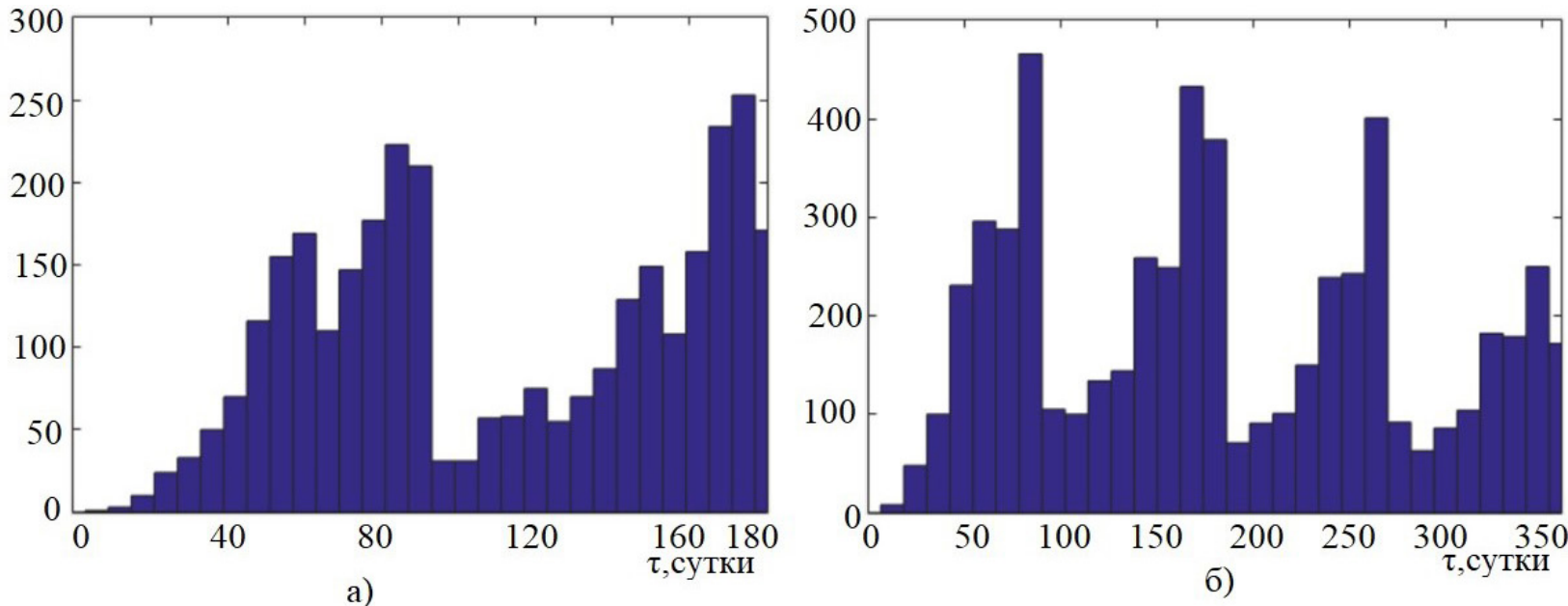

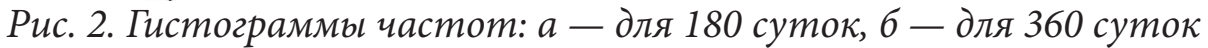

[Fig. 2. Histograms of frequencies: $a-$ for 180 days, $b-$ for 360 days] 
Оиенка показателей «Отказ в обслуживании» при организации ремонтных работ ...

360 суток. Гистограммы частот имеют вид «пилы» с периодом 90 суток. Причем левые стороны зубьев «пилы» изменяются немонотонно по возрастанию. Это связано с тем, что в данной модели в конце каждой декады идут платежи для выполнения текущих работ (2,334 млн. руб.), в конце месяца идут платежи для выполнения аварийных работ $(6,667$ млн. руб.), а в конце квартала (через 90 суток) идут платежи для выполнения капитальных работ, которые в деньгах самые большие (30 млн. руб.). Но самое главное, что в конце квартала выполняются все три платежа.

Отметим, что в работе [6] на основе выборки (4) проведены исследования по оценке ресурсно-затратного риска (2). В данной работе величины (3) в выборке (4) рассматриваются как моменты времени, когда происходит отказ в обслуживании оборудования. Обслуживание связано с ремонтными работами, а отказ происходит по причине отсутствия их финансового обеспечения. Элементы выборки (4) предложено рассматривать как наработки процесса ремонта оборудования, поэтому их предлагается обрабатывать, используя численные модели показателей надежности $[10,13]$. Модели называют численными (в отличии от аналитических), так как они обрабатывают числовую информацию, в нашем случае выборочные значения (4).

Показателями надежности являются:

1) численная вероятность безотказной работы;

2) численная средняя наработка;

3) численный гамма-процентный ресурс;

4) численная вероятность безотказной работы остаточного ресурса;

5) численный средний ресурс остаточного ресурса;

6) численный гамма-процентный остаточный ресурс.

\section{ЧИСЛЕННЫЕ МОДЕЛИ ПОКАЗАТЕЛЕЙ «ОТКАЗ В ОБСЛУЖИВАНИИ»}

Обозначим через $b$ - максимальное выборочное значение. Разобьем наблюдаемый интервал наработки $(0, b)$ узлами $t_{j}$ на $J$ интервалов одинаковой длины

$$
\Delta t=b / J ; t_{j}=j \cdot \Delta t, j=\overline{1, J}, t_{0}=0 ; t_{J}=b
$$

Обозначим через $n_{j}$ - объем выборочных значений, которые попадают в $j$-й интервал $\left(t_{j-1}, t_{j}\right)$. Тогда суммы относительных частот $m_{j}=\sum_{i=1} n_{i} / n, m_{J}=1$; обратные величины этих сумм относительно единицы равны

$$
k_{j}=1-m_{j} ; j=1, \ldots, J, k_{0}=1 .
$$

Величины (6) являются исходными данными для нахождения ступенчатой вероятности безотказной работы

$$
\begin{aligned}
& P^{c}(\tau)=k_{j}, t_{j-1} \leq \tau<t_{j}, j=\overline{1, J} \\
& P^{c}(0)=k_{0}=1 ; P^{c}(\tau)=0, t_{J-1} \leq \tau<t_{J} .
\end{aligned}
$$

Далее, заменив ступенчатую функцию (7) линейной, получим численную вероятность безотказной работы

$$
P_{r}(\tau)=\left\{\begin{array}{l}
1, \tau<0 \\
k_{j-1}+\left(\tau-t_{j-1}\right)\left(k_{j}-k_{j-1}\right) J / b, \\
\quad 0 \leq \tau<b \\
0, \tau \geq b,
\end{array}\right.
$$

где $t_{j-1} \leq \tau<t_{j}, j=\overline{1, J}$.

Используя зависимость между вероятностью безотказной работы и средней наработкой, найдем численную среднюю наработку

$$
\overline{t_{r}}=\int_{0}^{b} P_{r}(\tau) d \tau=\frac{b}{J}\left(0,5+\sum_{j=1}^{J} k_{j}\right) .
$$

Затем аналогично найдем численный гамма-процентный ресурс из уравнения

$$
P_{r}(\tau)=\gamma \rightarrow t_{r}(\gamma)=t_{j-1}+\frac{\left(\gamma-k_{j-1}\right)}{k_{j}-k_{j-1}} \cdot \frac{b}{J},
$$

где $k_{j-1} \geq \gamma>k_{j} \quad j=\overline{1, J}$; левая часть уравнения является функцией (8), а $\gamma-$ вероятность, для которой определяется гамма-процентный ресурс.

Остаточный ресурс оборудования [2] это время, определяемое по модели

$$
Y_{z}=T-z \text {. }
$$

Здесь $T-$ наработка оборудования как случайная величина; $z$ - время, которое оборудования уже проработало. В нашем случае наработка - это выборочные значения (4), а время

$$
\begin{gathered}
z=j \cdot b / J, j=\overline{1, J_{0}}, J_{0} \leq J-2 \\
y=i \cdot b / J, i=\overline{0, J-j} .
\end{gathered}
$$


Далее получим ступенчатую вероятность безотказной работы остаточного ресурса (11)

$$
P_{z}^{c}(y)=p_{j}(i)=k_{j+i} / k_{j}, p_{j}(0)=1 .
$$

Тогда численная вероятность безотказной работы остаточного ресурса с учетом формулы (12) имеет вид

$$
\begin{gathered}
\quad P_{z}^{r}(y)= \\
=\left\{\begin{array}{l}
1, y<0 \\
p_{j}(i-1)+\left(y-t_{i-1}\right)\left(p_{j}(i)-p_{j}(i-1)\right) \cdot J / b, \\
0, y \geq b-z
\end{array}\right.
\end{gathered}
$$

где

$$
t_{i-1} \leq y<t_{i}, t_{i}=i \cdot b / J, i=\overline{1, J-j} .
$$

С учетом формулы (13), численный средний остаточный ресурс имеет вид

$$
\tilde{y}_{z}=\int_{0}^{b-z} P_{z}^{r}(y) d y=\frac{b}{J}\left[0,5+\sum_{i=1}^{J-j} p_{j}(i)\right],
$$

а численный гамма-процентный остаточный ресурс определяется из уравнения, левая часть которого является функцией (13). Тогда

$$
\begin{gathered}
P_{z}^{r}(\tau)=\gamma \rightarrow \tilde{y}_{j}(\gamma)= \\
=t_{i-1}+\frac{\left(\gamma-p_{j}(i-1)\right)}{p_{j}(i)-p_{j}(i-1)} \cdot \frac{b}{J} .
\end{gathered}
$$

Здесь

$$
\begin{gathered}
p_{j}(i-1) \geq \gamma>p_{j}(i), \\
i=\overline{1, J-j}, \quad p_{j}(0)=1 .
\end{gathered}
$$

\section{ИССЛЕДОВАНИЕ ПОКАЗАТЕЛЕЙ «ОТКАЗ В ОБСЛУЖИВАНИИ» ОБОРУДОВАНИЯ}

Исследование показателей (8)-(15) проведем при следующих исходных данных:

1) число интервалов $J=30$;

2) вероятность гамма $\gamma=0,95$;

3) номер интервала для остаточного ресурса: $j=14$ и $j=17$;

4) максимальные выборочные значения (b) $=90,180,360$ (суток).

Виды законов распределений и значения числовых характеристик для интервалов времени и затратам приведены в табл. 1.

В табл. 2 приведены результаты расчетов показателей «Отказ в обслуживании», единица измерения сутки.

На рис. 3 приведена численная вероятность безотказной работы остаточного ресурса (13) при $b=180$ сут., $j=14$ ( $z=84$ сут.) и $j=17$ ( $z=102$ сут.), соответственно. На рис. 4 приведена численная вероятность безотказной работы (8) при $b=180$ сут.

Результаты исследования, приведенные в табл. 2 и на рис. 3-4, позволяют сделать следующие выводы:

\begin{tabular}{|c|c|c|c|}
\hline Показатели & 90 & 180 & 360 \\
\hline Численная средняя наработка & 66,740 & 113,018 & 187,133 \\
\hline Численный гамма-процентный ресурс & 33,889 & 43,587 & 50,350 \\
\hline Численный средний остаточный ресурс, $j=14$ & 28,355 & 56,613 & 91,548 \\
\hline Численный гамма-процентный остаточный ресурс, $j=14$ & 4,161 & 2,176 & 3,611 \\
\hline$z=$ & 42 & 84 & 168 \\
\hline Численный средний остаточный ресурс, $j=17$ & 22,683 & 50,985 & 80,336 \\
\hline Численный гамма-процентный остаточный ресурс, $j=17$ & 2,423 & 11,303 & 13,698 \\
\hline$z=$ & 51 & 102 & 204 \\
\hline
\end{tabular}

1) Созданное алгоритмическое и программное обеспечение позволяет количественно оценить показатели надежности, характеризующие отказ в обслуживании оборудования по причине отсутствия финансового обеспечения.

Таблииа 2. Оиенки показателей «Отказ в обслуживании», сут.

[Table 2. Evaluation indicators "Denial of Service» day] 
Оиенка показателей «Отказ в обслуживании» при организации ремонтных работ ...

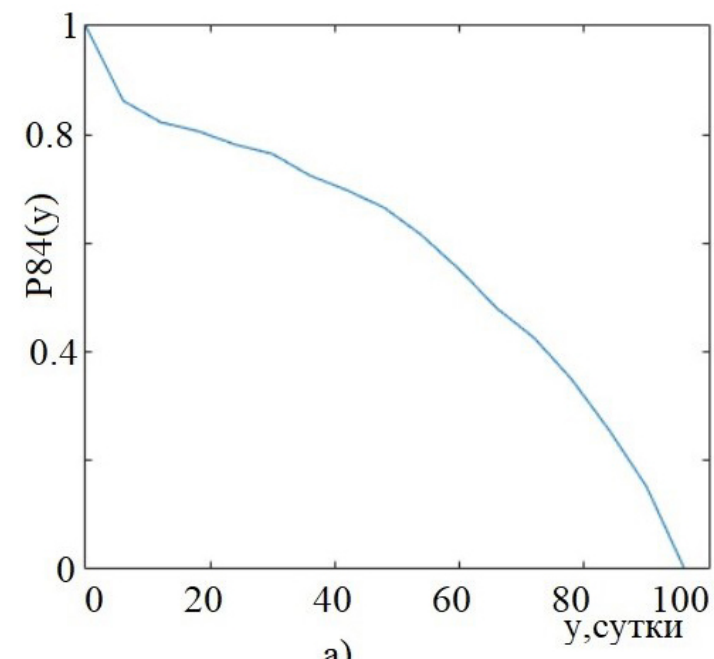

a)

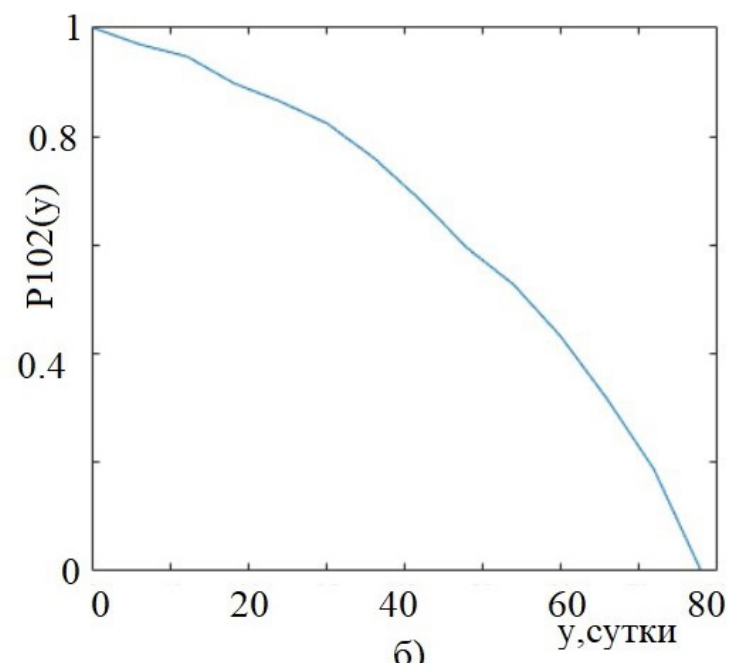

б)

Рис. 3. Численная вероятность безотказной работь остаточного ресурса $(b=180 \mathrm{cym}$.): $a-n p u j=14, \sigma-n p u j=17$

[Fig. 3. Numerical probability of failure-free operation of the remaining resource $(b=180$ days $): a-$ with $j=14, b-$ with $j=17$ ]

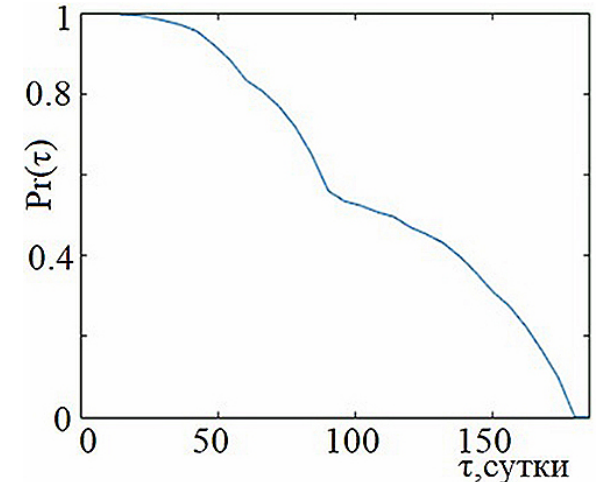

Рис. 4. Численная вероятность безотказной pаботы $(b=180 \mathrm{cym}$.

[Fig. 4. Numerical probability of failure-free operation $(b=180$ days $)]$

2) Учитывая характер гистограмм частот выборочных значений (4), вероятность безотказной работы (рис. 4) изменяется монотонно, но с разной скоростью. Это особенно заметно для времени 90 суток.

3) Характер гистограмм частот оказывает существенное влияние на показатели остаточного ресурса, особенно на гамма-процентный остаточный ресурс (табл. 2).

4) Пилообразный характер гистограмм частот определяется особенностью пополнения страхового фонда по видам ремонтных работ.

\section{ЗАКЛЮЧЕНИЕ}

Данное исследование является развитием работы [6], в которой для измерения эффективности организации ремонтных работ сложного оборудования предложено использовать точечную и интервальную оценки ресурсно-затратного риска. В данной работе предложено для этой задачи дополнительно использовать показатели надежности, характеризующие отказ в обслуживании оборудования по причине отсутствия финансового обеспечения. Введение этих показателей и их реализация является новизной работы.

Для этого создано алгоритмическое и программное обеспечение, позволяющее по выборочным значениям (4) количественно вычислять численные показатели, содержащие: вероятность безотказной работы, среднюю наработку, гамма-процентный ресурс, вероятность безотказной работы остаточного ресурса, средний остаточный ресурс; гамма-процентный остаточный ресурс. Показано, что гистограммы частот выборочных значений (4) имеют вид «пилы» с периодом 90 суток. Это определяется особенностью пополнения страхового фонда по видам ремонтных работ. Учитывая характер гистограмм частот, вероятность безотказной работы (рис. 4) изменяется монотонно, но с разной скоростью. Это особенно заметно для времени 90 суток. 
Характер гистограмм частот оказывает существенное влияние на показатели остаточного ресурса, особенно на гамма-процентный остаточный ресурс (табл. 2).

\section{КОНФЛИКТ ИНТЕРЕСОВ}

Авторы декларируют отсутствие явных и потенциальных конфликтов интересов, связанных с публикацией настоящей статьи.

\section{СПИСОК ЛИТЕРАТУРЫ}

1. Костюков, В. Н. Мониторинг безопасности производства / В. Н. Костюков - Москва: Машиностроение, 2002. - 224 с.

2. Краковский, Ю. М. Математические и программные средства оценки технического состояния оборудования / Ю. М. Краковский. - Новосибирск : Наука, 2006. - 228 с.

3. Крестин, Е. А. Диагностика машин и оборудования. / Е. А. Крестин, И. Е. Крестин. - Санкт-Петербург : Лань, 2016. - 376 с.

4. Калитенков, Н. В. Надежность и диагностика транспортного радиооборудования и средств автоматики / Н. В. Калитенков, В. С. Солодов. - Москва : МОРКНИГА, 2012. $-521 \mathrm{c}$.

5. Шишмарев, В. Ю. Диагностика и надежность автоматизированных систем / В. Ю. Шишмарев. - Москва : Academia, 2013. $352 \mathrm{c}$.

6. Краковский, Ю. М. Оценка ресурсно-затратного риска при организации ремонтных работ сложного роботизированного оборудования / Ю. М. Краковский, Н. А. Хоанг //
Вестник ВГУ. Серия: системный анализ и информационные технологии. - 2019. - № 4. C. 29-35.

7. Начигин, В. А. Формализация селективной технологии содержания инфраструктуры и страховой фонд / В. А. Начигин, Ю. М. Краковский // Мир транспорта. - 2015. - Т. 13, № 1. - С. 94-99.

8. Королев, В. Ю. Математические основы теории рисков / В. Ю. Королев, В. Е. Бенинг, С. Я. Шоргин - Москва: ФИЗМАТЛИТ, 2011. $-620 \mathrm{c}$.

9. Байхельт, Ф. Надежность и техническое обслуживание. Математический подход / Ф. Байхельт, П. Франкен. - Москва: Радио и связь, 1988. - 392 с.

10. Краковский, Ю. М. Моделирование перевозочного процесса железнодорожным транспортом: анализ, прогнозирование, риски / Ю. М. Краковский, С. К. Каргапольцев, В. А. Начигин; под ред. проф. Ю.М. Краковского. - Санкт-Петербург: «ЛИТЕО», 2018. $240 \mathrm{c}$.

11. Мак Томас. Математика рискового страхования / Томас Мак. Пер. с нем. - Москва: 3АО «Олимп-Бизнес», 2005. - 432 с.

12. Хоанг, Н. А. Моделирующая программа для исследования управления ремонтными работами сложного оборудования / Ю. М. Краковский, Н. А. Хоанг // Baikal Letter DAAD. - 2019. - № 1. - C. 64-72.

13. Хоанг, Н. А. Математическое обеспечение оценки показателей надежности технологического оборудования / Ю. М. Краковский, Н. А. Хоанг // Актуальные вопросы аграрной науки. - 2018. - № 27. - С. 51-57.

Краковский Юрий Мечеславович - д-р техн. наук, проф., профессор кафедры «Информационные системы и защита информации», Иркутский государственный университет путей сообщения.

E-mail: 79149267772@yandex.ru

ORCID iD:https://orcid.org/0000-0003-4876-0618

Хоанг Нгок Ань - аспирант кафедры информатики и математического моделирования, Иркутский государственный аграрный университет имени А. А. Ежевского.

E-mail: hoangngocanhmta@gmail.com

ORCID iD:https://orcid.org/0000-0003-2798-5551 


\title{
ASSESSMENT OF THE INDICATORS OF "SERVICE UNAVAILABILITY" WHEN ORGANIZING REPAIR WORKS OF COMPLEX EQUIPMENT
}

\author{
(c) 2020 Y. M. Krakovsky ${ }^{\bowtie 1}$, N. A. Hoang ${ }^{2}$ \\ ${ }^{1}$ Irkutsk State Transport University \\ 15, Chernyshevsky Str., 664074 Irkutsk, Russian Federation \\ ${ }^{2}$ Irkutsk State Agrarian University named after A. A. Ezhevsky \\ 1/1, Molodezhny settlement, 664038 Irkutsk district, Irkutsk region, Russian Federation
}

\begin{abstract}
The article discusses the organization of repair works of complex equipment. To organise such works, an insurance fund is necessary which has two functions: it accumulates money paid over different time intervals to perform various types of repair works, and, when necessary, provides financing to pay for these works. The mathematical description of the state of the insurance fund is based on a random risk process used in risk theory. This approach allowed us to introduce numerical models of reliability indicators characterizing service unavailability in relation to equipment maintenance. The service is connected with repair works and the lack of availability occurs due to the lack of financial support for them. Numerical models of reliability indicators include: the probability of failure-free operation, the average operating time, the gamma-percent resource, the probability of failure-free operation of the residual resource, the average residual resource; the gamma percent residual life. To study these indicators, we designed a programme which, using an event based approach, creates the necessary sample values and then processes them numerically. The simulation programme is able to be "tuned" to various sources of data, which provides us the opportunity to conduct research using a wide range of data sources.
\end{abstract}

Keywords: complex equipment, random risk process, reliability indicators, simulation modelling.

\section{CONFLICT OF INTEREST}

The authors declare the absence of obvious and potential conflicts of interest related to the publication of this article.

\section{REFERENCES}

1. Kostyukov V. N. Monitoring the safety of production. Moscow. Mechanical Engineering, 2002. $224 \mathrm{p}$.

2. Krakovsky Y. M. Mathematical and software tools for assessing the technical state of equipment. Novosibirsk: Nauka, 2006. 228 p.

3. Krestin E. A., Krestin E.A. and Christening I. E. Diagnostics of machinery and equipment. St. Petersburg: Doe, 2016. 376 p.

4. Kalitenkov N. V., Solodov V.S. Reliability and diagnostics of transport radio equipment and automation equipment. Moscow: MORKNIG, 2012. $521 \mathrm{p}$.

5. Shishmarev V. Yu. Diagnostics and reliability of automated systems. Moscow: Academia, 2013. $352 \mathrm{p}$.

6. Krakovsky Y. M., Hoang N. A. Assessment of resource-cost risk in the organization of repair work of complex robotic equipment. Proceedings of VSU, Series: system analysis and information technology.2019. No. 4. P. 29-35.

7. Nachigin V. A., Krakovsky Y. M. Formalization of selective technology for maintaining infrastructure and insurance fund. World of Transport.2015.T. 13.No. 1. pp. 94-99.

8. Beichelt F., Franken P. Reliability and maintenance. The mathematical approach. Moscow: Radio and Communications, 1988. 392 p.

Krakovsky Yuri M.

e-mail: 79149267772@yandex.ru 
9. Krakovsky Y. M., Kargapoltsev S. K., Nachigin $V$. A. Modeling the transportation process by rail: analysis, forecasting, figs. under the editorship of prof. Y. M. Krakovsky. - St. Petersburg : LITEO, 2018. 240 p.

10. Mack Thomas. Mathematics of Risk Insurance. Moscow : CJSC Olimp-Business, 2005. $432 \mathrm{p}$.

11. Korolev V. Yu., BeNing V. E., Shorgin S. Ya. Mathematical foundations of risk theory. Moscow: FIZMATLIT, 2011. 620 p.
12. Hoang N. A., Krakovsky Y. M. Modeling program for the study of the management of repair work of complex equipment. Baikal Letter DAAD. 2019. No. 1. pp. 64-72.

13. Hoang N. A., Krakovsky Y. M. Mathematical support for assessing the reliability indicators of technological equipment. Actual problems of agricultural science. - 2018. No. 27. P. 51-57.

Krakovsky Yuri M. - DSc in Technical Sciences, Professor, Department of Information Systems and Information Security, Irkutsk State Transport University.

E-mail: 79149267772@yandex.ru

ORCID iD:https://orcid.org/0000-0003-4876-0618

Hoang Ngoc A. - postgraduate student, Department of Informatics and Mathematical Modelling, Irkutsk State Agricultural University named after A. A. Ezhevsky.

E-mail: hoangngocanhmta@gmail.com

ORCID iD:https://orcid.org/0000-0003-2798-5551 